\title{
Discovering and dealing with the unknown aspects of Colletotrichum
}

\author{
RS Jayawardena ${ }^{1,2}$ *, JY Yan ${ }^{1}$, L Cai ${ }^{3}$, BD Shenoy ${ }^{4}$ and G Sharma ${ }^{5}$ \\ ${ }^{1}$ Institute of Plant and Environment Protection, Beijing Academy of Agriculture and Forestry Sciences, Beijing \\ 100097, People's Republic of China \\ ${ }^{2}$ Center of Excellence in Fungal Research, Mae Fah Luang University, Chiang Rai 57100, Thailand \\ ${ }^{3}$ State Key Laboratory of Mycology, Institute of Microbiology, Chinese Academy of Sciences, Beijing, 100101, \\ China \\ ${ }^{4}$ CSIR-National Institute of Oceanography Regional Centre, \#176, Lawson's Bay Colony, Visakhapatnam - 530017, \\ India \\ ${ }^{5}$ Regional Centre for Biotechnology, NCR Biotech Science Cluster, Faridabad-Gurgaon Expressway, Faridabad- \\ 121001, Haryana (NCR Delhi), India \\ *email-ruvi.jaya@yahoo.com
}

RS Jayawardena, JY Yan, L Cai, BD Shenoy, G Sharma 2016 - Discovering and dealing with the unknown aspects of Colletotrichum. Mycosphere 7(8) 1074-1075, Doi $10.5943 / \mathrm{mycosphere} / \mathrm{si} / 2 \mathrm{c} / 10$

Colletotrichum species have diverse life styles as pathogens, endophytes and saprobes, and have attracted great attention from mycologists and plant pathologists, since the introduction by Corda in 1831. This genus was also a main source of confusion among taxonomists due to the belief that species were host-specific. After several morphological revisions, a special issue on Colletotrichum published in Fungal Diversity in 2009 provided a background to series of studies that used molecular phylogeny, together with morphology, to resolve species. Recent polyphasic approaches have helped to define species in the genus, especially in resolving the species complexes. In 2012, a special issue of Studies in Mycology resolved species in three major species complexes and establshed a list of accepted species for this genus. Many other studies have also been conducted in resolving species complexes and identifying species on various hosts.

Although there have been great improvements in the taxonomy of Colletotrichum, at present there is no consensus as to which loci should be used to infer phylogeny. As morphological characters and ITS sequence data alone do not provide significant resolution for distinguishing closely related species, it has become important to use a secondary barcodes to identify Colletotrichum species.

The present issue of Mycosphere comprises of two mycosphere essays and seven papers on Colletotrichum, that represent current interest and future perspectives. In the first essay Jayawardena et al. (Paper 1) emphasises the importance of accurately naming Colletotrichum species. Sharma and Shenoy (Paper 2) discuss the methods prevalent in Colletotrichum 
systematics and also provide suggestions towards developing a stable and reliable classification system. This paper also emphasises the need for a secondary barcodes to resolve Colletotrichum species. Huang et al. (Paper 3) assess the genetic diversity of the grape ripe rot pathogen $C$. gloeosporioides using SRAP markers. Hou et al. (Paper 4) describe two new holomorphic species of Colletotrichum from China and Japan. Whole genome re-sequencing of $C$. gloeosporioides was carried out by Huang et al. (Paper 5), which provides the first comprehensive trancriptomic resource available for this species. Jayawardena et al. (Paper 6) studied species associated with strawberry anthracnose in China, providing an update of the causal agents, and revealing a new species. The second essay by Jayawardena et al. (Paper 7) emphasises the importance of Colletotrichum species in biological control, as well as its uses in industrial appications. Lei et al. (Paper 8) studied the species causing grape ripe rot in southern China and provide reports of two new host records. In the final paper Jayawardena et al. (Paper 9) provide an updated list of species in Colletotrichum together with their habit, host and geographic distribution, phylogenetic position, their sexual morphs and industrial uses if applicable. It also suggests the genes and combination of genes that can be used for identification of the various species complexes. 